\title{
Multi-GPU-based Swendsen-Wang multi-cluster algorithm for the simulation of two-dimensional $q$-state Potts model
}

\author{
Yukihiro Komura $^{\mathrm{a}}$, Yutaka Okabe ${ }^{\mathrm{a}}$ \\ ${ }^{a}$ Department of Physics, Tokyo Metropolitan University, Hachioji, Tokyo 192-0397, Japan
}

\begin{abstract}
We present the multiple GPU computing with the common unified device architecture (CUDA) for the Swendsen-Wang multi-cluster algorithm of two-dimensional (2D) $q$-state Potts model. Extending our algorithm for single GPU computing [Comp. Phys. Comm. 183 (2012) 1155], we realize the GPU computation of the Swendsen-Wang multi-cluster algorithm for multiple GPUs. We implement our code on the large-scale open science supercomputer TSUBAME 2.0, and test the performance and the scalability of the simulation of the 2D Potts model. The performance on Tesla M2050 using 256 GPUs is obtained as 37.3 spin flips per a nano second for the $q=2$ Potts model (Ising model) at the critical temperature with the linear system size $L=65536$.

Keywords: Monte Carlo simulation, cluster algorithm, Ising model, parallel computing, Multi-GPU
\end{abstract}

\section{Introduction}

High performance computing accelerates advances in science. Recently the use of general purpose computing on graphics processing unit (GPU) is a hot topic in computer science. Drastic reduction of processing times can be realized in scientific computations. Using the common unified device architecture (CUDA) released by NVIDIA, it is now easy to implement algorithms on GPU using standard $\mathrm{C}$ or $\mathrm{C}++$ language with CUDA specific extension.

Preprint submitted to Elsevier

October 24, 2018 
Multiple GPU computing is performed for accommodating larger-scale problems beyond the capacity of video memory on a single GPU and for speedup of the fixed problem pursuing strong scalability. Multiple GPU computing requires GPU-level parallelization. A large-scale open science supercomputer TSUBAME 2.0 is available at the Tokyo Institute of Technology. TSUBAME 2.0 consists of 4224 NVIDIA Tesla M2050 GPUs as a total, and the theoretical peak performance reaches 2.4 PFLOPS

Studying spin models is a basic problem in statistical physics. At the same time the Monte Carlo simulation of spin models has been served as a benchmark of newly developed technology. Sublattice decomposition was used in the Metropolis-type 11] Monte Carlo simulation of spin models in vector computer, and the same idea was employed in the GPU-based calculation of the Ising model by Preis et al. 22, 3]. The GPU-based calculation of the multispin coding of the Metropolis-type Monte Carlo simulation was proposed by Block et al. [4]. There, they argued the multiple GPU calculation in order to overcome the memory limitations of a single GPU, and they found that for large systems, the computation time scales nearly linearly with the number of GPUs.

The Metropolis-type single-spin-flip algorithm often suffers from the problem of slow dynamics or the critical slowing down. To overcome difficulty, a cluster flip algorithm was proposed by Swendsen and Wang [5]. In the SwendsenWang (SW) algorithm, all the spins are partitioned into clusters; thus, the SW algorithm is called the multi-cluster algorithm. Wolff [6] proposed another type of cluster algorithm, that is, a single-cluster algorithm, where only a single cluster is generated, and the spins of that cluster are updated.

The high performance computing using GPUs are highly desirable for Monte Carlo simulations with cluster flip algorithms. Recently, some attempts have been reported along this line on a single GPU. The present authors [7] have proposed the GPU-based calculation with CUDA for the Wolff single-cluster algorithm, where parallel computations are performed for the newly added spins in the growing cluster. Hawick et al. 8] have studied the CUDA implementation of the Wolff algorithm using a modified connected component labeling 
for the assignment of the cluster. They put more emphasis on the hybrid implementation of Metropolis and Wolff updates and the optimal choice of the ratio of both updates. Weigel [9] has studied parallelization of cluster labeling and cluster update algorithms for calculations with CUDA. He realized the SW multi-cluster algorithm by using the combination of self-labeling algorithm and label relaxation algorithm or hierarchical sewing algorithm. Quite recently, the present authors 10] have proposed the GPU calculation with CUDA for the SW multi-cluster algorithm by using the two connected component labeling algorithms, the algorithm by Hawick et al. 11] and that by Kalentev et al. 12], for the assignment of clusters. The computational speed for the $q=2$ Potts model on NVIDIA GeForce GTX580 was 12.4 times as fast as the computational speed on a current CPU core, Intel(R) Xeon(R) CPU W3680.

Finite-size scaling works well for the systems which undergo second-order phase transition; therefore numerical studies on smaller systems can lead to the understanding of phase transition with the help of finite-size scaling. On the other hand, it is a different story for the Kosterlitz-Thouless (KT) transition 13], where logarithmic behavior is essential. Large scale simulations are needed for the systems which show the KT transition.

In this paper, we present the multiple GPU calculation with CUDA for the SW multi-cluster algorithm of the two-dimensional (2D) $q$-state Potts model. This is an extension of the GPU-based calculation on a single GPU presented in Ref. [10]. We test the performance of our algorithm for the 2D q-state Potts model on the multiple GPUs of TSUBAME 2.0. The rest of the paper is organized as follows. In section 2, we briefly review the GPU-based calculation of the SW multi-cluster algorithm on a single GPU described in Ref. [10]. In section 3, we present the idea and implementation of GPU-based calculation extended to the case of multiple GPUs. In section 4, we test the performance of multiple GPU calculation on TSUBAME 2.0. The summary and discussion are given in section 5 . 


\section{Single GPU computing of the Swendsen-Wang cluster algorithm}

Swendsen and Wang proposed a Monte Carlo algorithm of multi-cluster flip [5]. To explain the SW algorithm, we use the $q$-state Potts model whose Hamiltonian is given by

$$
\mathcal{H}=-J \sum_{<i, j>}\left(\delta_{S_{i}, S_{j}}-1\right), \quad S_{i}=1,2, \cdots, q,
$$

and this corresponds to the Ising model for $q=2$. Here, $J$ is the coupling and $S_{i}$ is the Potts spin on the lattice site $i$. The summation is taken over the nearest neighbor pairs $\langle i, j\rangle$. Periodic boundary conditions are employed.

There are three main steps in the SW algorithm: (1) Construct a bond lattice of active or non-active bonds depending upon the temperature. (2) The active bonds partition the spins into clusters which are identified and labeled using a cluster-labeling algorithm. (3) All spins in each cluster are set randomly to one of $q$.

For an efficient cluster-labeling algorithm, the Hoshen-Kopelman algorithm 14], which was first introduced in context of cluster percolation, is often used. In the Hoshen-Kopelman cluster-labeling algorithm, integer labels are assigned to each spin in a cluster. Each cluster has its own distinct set of labels. The proper label of a cluster, which is defined to be the smallest label of any spin in the cluster, is found by the following function. The array label is used, and if label is a label belonging to a cluster, label [label] is the index of another label in the same cluster which has a smaller value if such a smaller value exists. The proper label for the cluster is found by evaluating label [label] repeatedly.

Since the calculations of the step of active bond generation and the step of spin flip are done independently on each site, these steps are well suited for parallel computation on GPU. On the other hand, in the step of cluster labeling the assignment of label of cluster is done on each site piece by piece sequentially; thus the cluster-labeling algorithm such as the Hoshen-Kopelman algorithm cannot be directly applied to the parallel computation on GPU.

The present authors [10] have proposed the calculation on a single GPU 
with CUDA for the SW multi-cluster algorithm by using the cluster-labeling algorithm appropriate for the parallel computation using GPU. The recently proposed algorithm, the "Label Equivalence" algorithm, by Hawick et al. [11] together with its refined version by Kalentev et al. [12] were used in Ref. [10]. Since the algorithm by Kalentev et al. [12] is superior to that by Hawick et al. [11], and we extend it to the multiple GPU computing, we briefly explain the "Label Equivalence" algorithm by Kalentev et al. [12].

The procedure of their algorithm [12], which was schematically illustrated in Fig. 2 of Ref. [10], consists of the two kernel functions, that is, scanning function and analysis function. The scanning function compares the label of each site with that of the nearest-neighbor sites when the bond between each site and the nearest-neighbor site is active. If the label of the nearest-neighbor site is smaller than the label of that site, the label variable with the label number, label[label], is updated to the smallest one. The analysis function resolves the equivalence chain of the label obtained in the scanning function; the label variable is updated from the starting site to the new site, which is similar to the method of the Hoshen-Kopelman algorithm. Each thread tracks back the label variable until the label variable, label, remains unchanged. In the "Label Equivalence" algorithm, the loop including two kernel functions is iterated up to the point when the information of the labeling needs no more process of scanning function. The number of iterations for the $q=2$ Potts model at the critical temperature is about 8.6 on average for $4096 \times 4096$ systems.

\section{Multiple GPU computing of the Swendsen-Wang cluster algorithm}

To use multiple GPUs, the data communication between GPUs is necessary. However it is not able to communicate directly between GPUs when many GPUs are used. The data transfers on three levels are needed, that is, the data transfer from GPU to CPU, that between CPUs and that from CPU to GPU. We use the message passing interface (MPI) library for the communication between CPUs, and we run the same process number for MPI as the GPU number. These data communications reduce the computation efficiency 
(a)

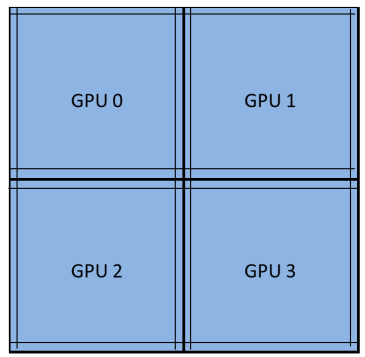

(b)

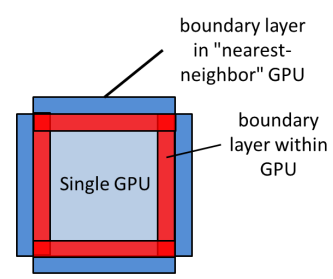

Figure 1: (a) The $2 \times 2$ super-lattice structure for 4 GPUs. (b) The information on a single GPU. It has the arrays to preserve the data of surrounding boundary layers and to transfer the data of boundary layers.

of multiple GPUs. Thus, hiding the cost of data communication by overlapping communication and computation is desirable such as in Ref. [15, 16, 17].

We arrange the total lattice with a super-lattice structure using multiple GPUs. Each GPU has the information of spins on a sub-lattice together with the arrays to preserve the data of surrounding boundary layers and to transfer the data of boundary layers. We illustrate the case of $2 \times 2$ super-lattice structure using 4 GPUs in figure 1 The present super-lattice and sub-lattice structures are similar to the structures employed in [4].

The calculation of active bond generation is performed in the same manner as that for a single GPU only with the copy of boundary layers. However, the calculation of cluster labeling needs extra process. Within each GPU, we follow the cluster labeling method of Kalentev [12], which consists of the scanning kernel function and analysis kernel function. But we have to check whether the sites of the different sub-lattices (GPUs) belong to the same cluster. We employ a two-stage process of cluster labeling. After the cluster labeling within each GPU is finished, we check the bond between the sites of "nearestneighbor" GPU; that is, the inter-GPU scanning function. For this purpose, we use two variables to describe the labeling of the cluster. One is a temporal variable label to make a cluster labeling within each GPU. The other variable label_total_lattice has the information on the labeling over the total lattice; 
it is described by the combination of the GPU number and the labeling within that GPU. When the labeling of the "nearest-neighbor" GPUs is compared, the variable label_total_lattice[label] is updated to the label_total_lattice with smaller GPU number. Then, the equivalence chain is resolved in a similar method to the analysis kernel function within a single GPU; that is, the inter-GPU analysis function.

To accelerate the computational speed, we arrange the process of the interGPU cluster labeling such that only the calculation of boundary layers in the sub-lattice is made. In the inter-GPU scanning function, we make parallel calculation for the boundary layers in "nearest neighbor" GPU. In the inter-GPU analysis function, we only update the variable label_total_lattice[label] of the arrays to transfer the data of boundary layers within GPU. After the iteration of the inter-GPU cluster labeling ends, each GPU updates the variable label_total_lattice[label] of the total sub-lattice. By using this method, the amount of calculation in the inter-GPU cluster labeling is proportional to $4 N_{x}$ instead of $N_{x} \times N_{x}$ for the linear system size of each GPU $N_{x}$.

The step of spin update is not straightforward. If we try to choose a new spin state after the step of cluster labeling, we face with the problem that we have to copy all the information of new spin states. To overcome this difficulty, we include the information on new spin state in the process of cluster labeling. Because of distributed memory, we cannot directly access the spin state of different GPU. This problem of multi-cluster flip Monte Carlo is not a local one. The information of boundary layer is not enough. The situation is different from the case of partial differential equation, for example.

The informations of new spin state and active bonds with up and left directions are included in a single word label or label_total_lattice to save memory and access time. The data structure is shown in figure 2 . The label is defined as an unsigned integer type of 32 bit, and the label_total_lattice is defined as an unsigned long long integer type of 64 bit. We note that the new spin state in label_total_lattice is that for the labeling in the total lattice, and is not necessarily the same as the temporal new spin state in label. 


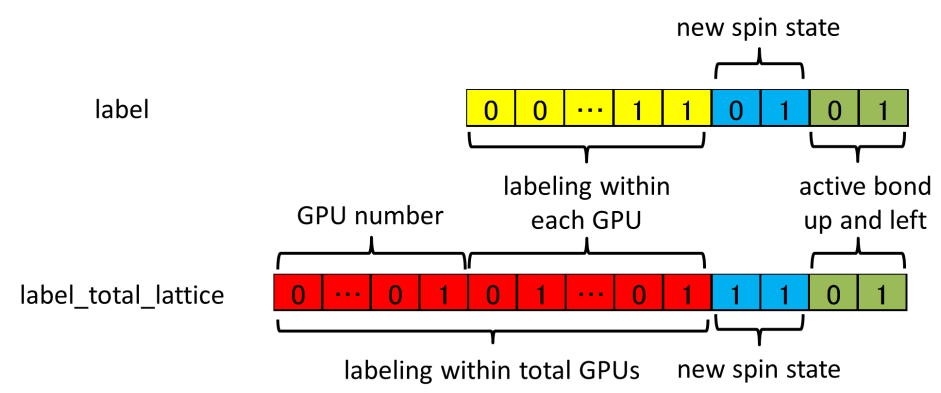

Figure 2: The data structures of two variables for labeling. A temporal variable "label" is used for saving the labeling within each GPU, and a variable "label_total_lattice" is used for saving the labeling information on the total lattice. The informations on new spin state and active bonds of up and left directions are included in a single word. The case of 4-state spin is shown as an example.

The data communication between GPUs is needed only in the step of cluster labeling. In order to improve the performance of the multiple-GPU computing, we use an overlapping technique between communication and computation. We pick up the data which are not referenced in the running kernel function. The scanning kernel function does not reference the data for the transfer of boundary layers within each GPU, and the analysis kernel function does not reference the data of boundary layers in "nearest-neighbor" GPU. The scanning kernel function is launched as the stream 0 ; the data for the transfer of boundary layers within each GPU are transferred from the global memory to host memory using the asynchronous Application Programming Interface (API) as the stream 1. In a similar way, the analysis kernel function is launched as the stream 0 , and the data of boundary layers in "nearest-neighbor" GPUs are transferred from the host memory to global memory using the asynchronous API as the stream 1. We use the technique of overlapping communication and computation for both the cluster labeling within each GPU and the inter-GPU cluster labeling. We note that it needs two loops of scanning function and analysis function to reflect the update of data of boundary layers in "nearest-neighbor" GPU. To terminate the loop in the cluster labeling, we check that the termination conditions for all GPUs are satisfied. To do this, the "flag"s for the termination condition 


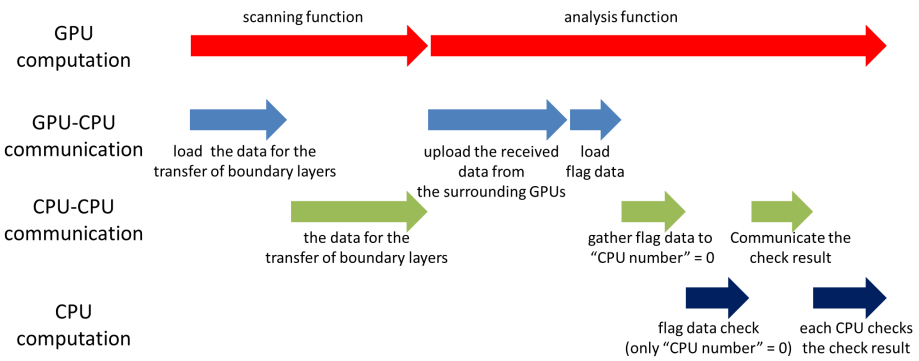

Figure 3: The procedure of overlapping data transfer and computation at the step of cluster labeling. During the computation in the scanning function, the data of boundary layers are transferred from each GPU to each CPU, and then the data are transferred from each CPU to the surrounding CPUs by MPI command. During the computation in the analysis function, the received data from the surrounding CPUs are uploaded from each CPU to each GPU. To check the termination conditions in all GPUs, the "flag"s of termination condition for all GPUs are gathered in one CPU, and that CPU checks the total termination condition and communicates the result of termination to all CPUs.

are gathered in one CPU during the implementation of analysis kernel function. And that CPU checks the total termination condition and the check result of termination is communicated to all CPUs by MPI command. The procedure of overlapping data transfer and computation at the step of cluster labeling is schematically shown in figure 3 ,

We finally note that we use a linear congruential random generator which was proposed by Preis et al. 2] when random numbers are generated.

\section{Results}

We have implemented our code of the multiple GPU computing for the 2D $q$-state Potts models on TSUBAME 2.0 with NVIDIA Tesla M2050 GPUs using CUDA 4.0 and openMPI 1.4.2. We have checked our code by plotting the temperature dependence of the moment ratio of magnetization as in Fig. 4 of Ref. [10], for example. We here present the data of the performance for the $q=2$ Potts model (Ising model) at the critical temperature, $T_{c} / J=$ $1 / \ln (1+\sqrt{2})=1.1346$. The average amount of spin flips per a nano second at the critical temperature for the $q=2$ Potts model is tabulated in table1. There, the average amounts of spin flips per a nano second for only spin updates and 
that including the measurement of energy and magnetization are given. The size of sub-lattice for each GPU is fixed as $4096 \times 4096$, and we increase the number of GPUs. The linear system sizes for the total lattice are $L=4096,8192$, 16384, 24576, 32768, 49152 and 65536 for the number of GPUs as 1, 4, 16, 36, 64,144 and 256, respectively.

\begin{tabular}{clc}
\hline $\begin{array}{c}\text { system size of total lattice } L \\
\text { (the number of GPUs })\end{array}$ & update only & $\begin{array}{c}\text { update } \\
+ \text { measurement }\end{array}$ \\
\hline $4096($ ( GPU) & 0.232 & 0.206 \\
$8192($ 4 GPU) & 0.813 & 0.729 \\
$16384($ 16 GPU) & 2.927 & 2.657 \\
$24576($ 36 GPU $)$ & 6.149 & 5.582 \\
$32768($ 64 GPU $)$ & 10.36 & 9.599 \\
$49152(144 \mathrm{GPU})$ & 22.67 & 20.53 \\
$65536(256 \mathrm{GPU})$ & 37.30 & 33.85 \\
\hline
\end{tabular}

Table 1: Average amount of spin flips per a nano second at $T_{c}$ for the $q=2$ Potts model with multiple GPUs. The numbers of spin flips per a nano second for only spin updates and that including the measurement of energy and magnetization are given.

To compare with the code for single GPU computing, we run the code in Ref. 10] with NVIDIA Tesla M2050. The average amount of spin flips per a nano second at $T_{c}$ for the $q=2$ Potts model of size $4096 \times 4096$ is 0.26 . Thus, the calculation speed of the code for multi GPU computing in this paper, 0.23 spin flips per a nano second, is nearly equal to the calculation speed of the code for single GPU computing. And, the performance of our code for multi GPU computing increases with the number of GPUs. The best performance is 37.3 spin flips per a nano second for the system with $L=65536$ by using 256 GPUs.

Next we examine the scalability of our code with changing the number of GPUs at the critical temperature for the $q=2$ Potts model. We give the double logarithmic plot of the average amount of spin flips per a nano second as a function of the number of GPUs in figure 4. For the size of sub-lattice of each GPU, not only the data for $4096 \times 4096$ but also those for $2048 \times 2048$ and $1024 \times 1024$ are plotted. The dependences on the number of GPUs with fixing the total linear system size as $L=4096,8192,16384,32768,65536$ are shown by using the same mark in figure 4 . Since the number of iterations at the step of 


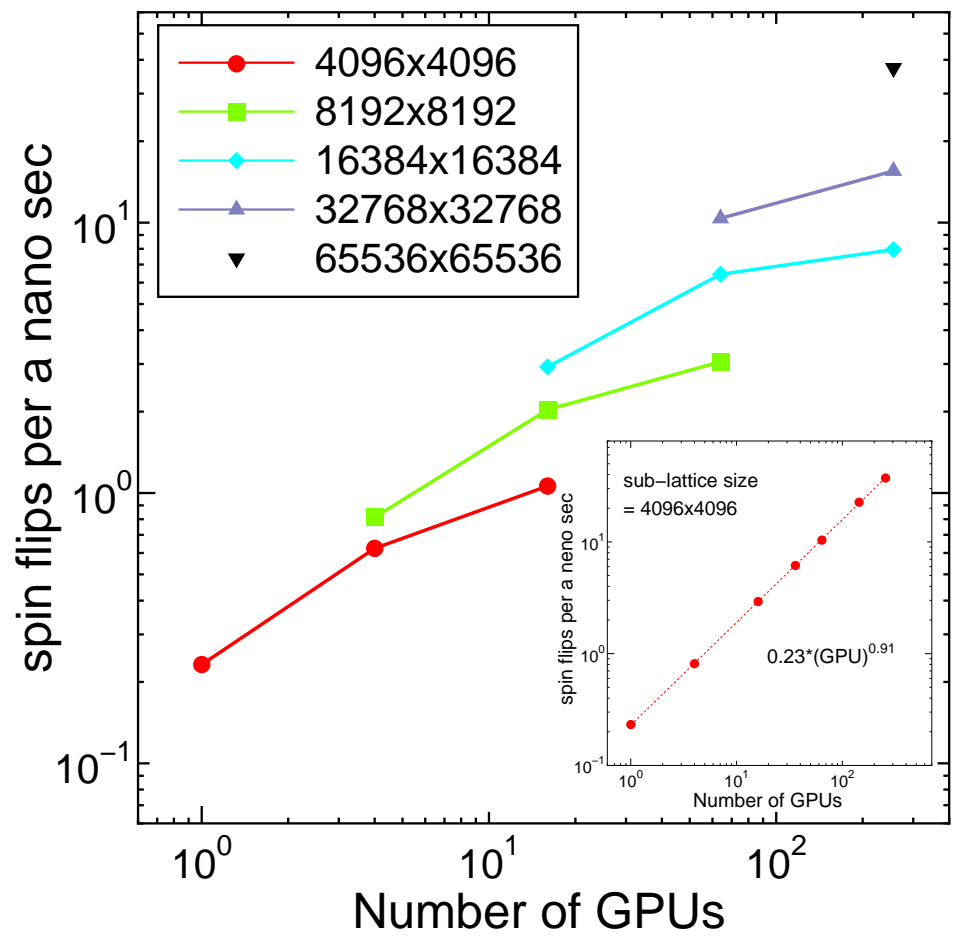

Figure 4: Plot of spin flips per a nano second with changing the number of GPUs at the critical temperature for the $q=2$ Potts model. The data for the sub-lattice size for each GPU as $1024 \times 1024,2048 \times 2048$ and $4096 \times 4096$ are plotted. The same mark is used for plotting the data for fixing the linear system size of the total lattice as $L=4096,8192,16384,32768$ and 65536. In the inset the values of fixing the sub-lattice size as $4096 \times 4096$ are plotted as a function of the number of GPUs. The best-fitted curve to get the power dependence is given by the dotted line. 
inter-GPU cluster labeling increases with the increase of the number of GPUs, the efficiency of the average spin flips per a nano second slightly decreases with the decrease of the sub-lattice size. We can see this tendency in Figure 4 . In the inset the dependence on the number of GPUs with fixing the sub-lattice size as $4096 \times 4096$ is shown. We see that the performance of our code increases as a power of the number of GPUs. The coefficient of the power is estimated as 0.91 by the best-fitted curve shown in the inset. Since it takes extra time to diffuse the label number from one GPU to all GPUs, the perfect weak scalability, that is, the power of 1.0, is not achieved, but we can say the weak scalability is well satisfied for our code.

\section{Summary and discussion}

Extending our algorithm for single GPU computing proposed in Ref. [10], we have developed the calculation of the SW multi-cluster algorithm for multiple GPU computing. In the step of cluster labeling, we employ a two-stage process; that is, we first make the cluster labeling within each GPU, and then the interGPU labeling is performed with reference to the label on the first-stage. We use some trick for the step of spin update; we include the information on new spin state in the process of cluster labeling. A special attention is paid to hide the communication overhead. We use the technique of overlapping communication and computation. And, we arrange the process of the inter-GPU cluster labeling such that only the calculation of boundary layers in the sub-lattice is made in order to gain the computational speed. The multiple GPU computing can be used not only for higher performance but also for accommodating larger-scale problems beyond the capacity of video memory on a single GPU. Thus the algorithm proposed in this paper permits us to deal with the calculation of the SW multi-cluster Monte Carlo simulation for large systems.

We have implemented our code of the SW algorithm for the 2D $q$-state Potts model on the large-scale open science supercomputer TSUBAME 2.0. We have tested the performance of our code for the $q=2$ Potts model. As a result, the average amount of spin flips per a nano second by using 256 GPUs is 37.3 at the 
critical temperature for the total linear system size $L=65536$. And we have examined the scalability of our code at $T_{c}$ with changing the number of GPUs. The average amount of spin flips per a nano second is proportional to a power of the number of GPUs with the power being 0.91. We have confirmed both the strong and weak scalabilities.

We have shown in this paper that the SW multi-cluster algorithm is well suited for multiple GPU computing, although an early study suggested that the SW multi-cluster algorithm is not suitable for the multiple parallel computation 18]. We emphasize that the implementation of the cluster algorithm for multiple GPU computing is highly efficient because the convergence of the cluster algorithm is several orders of magnitude faster than the single-spin flip Monte Carlo simulation near the critical point for large systems. This computation is easily extended to continuous spin models, such as the classical XY model, using the Wolff's idea of embedded cluster [6]. It is interesting to perform large scale simulations for the 2D classical XY model, which undergoes the KT transition, as mentioned in Introduction. The multiple GPU calculation for the cluster algorithm of the classical XY model in connection with the probability-changing cluster algorithm [19, 20] is now in progress.

\section{Acknowledgment}

We thank Takayuki Aoki for valuable discussions. The computation of this work has been done using the large-scale open science supercomputer TSUBAME 2.0 at the Tokyo Institute of Technology. This work was supported by a Grant-in-Aid for Scientific Research from the Japan Society for the Promotion of Science.

\section{References}

[1] N. Metropolis, A.W. Rosenbluth, M.N. Rosenbluth, A.H. Teller, E. Teller, Equation of State Calculations by Fast Computing Machines, J. Chem. Phys. 21 (1953) 1087-1092. 
[2] T. Preis, P. Virnau, W. Paul, J.J. Schneider, GPU accelerated Monte Carlo simulation of the 2D and 3D Ising model, J. Comp. Phys. 228 (2009) 44684477.

[3] T. Preis, GPU-computing in econophysics and statistical physics, Eur. Phys. J. Special Topics 194 (2011) 87-119.

[4] B. Block, P. Virnau, T. Preis, Multi-GPU accelerated multi-spin Monte Carlo simulations of the 2D Ising model, Comp. Phys. Comm. 181 (2010) 1549-1556.

[5] R.H. Swendsen, J.S. Wang, Nonuniversal critical dynamics in Monte Carlo simulations, Phys. Rev. Lett. 58 (1987) 86-88.

[6] U. Wolff, Collective Monte Carlo Updating for Spin Systems, Phys. Rev. Lett. 62 (1989) 361-364.

[7] Y. Komura, Y. Okabe, GPU-based single-cluster algorithm for the simulation of the Ising model, J. Comp. Phys. 231 (2012) 1209-1215.

[8] K. A. Hawick, A. Leist, D. P. Playne, Cluster and Fast-update Simulations of Regular and Rewired Lattice Ising Models Using CUDA and Graphical Processing Units, Technical Report CSTN-104 (2010).

[9] M. Weigel, Connected component identification and cluster update on GPU, Phys. Rev. E 84 (2011) 036709.

[10] Y. Komura, Y. Okabe, GPU-based Swendsen-Wang multi-cluster algorithm for the simulation of two-dimensional classical spin systems, Comp. Phys. Comm. 183 (2012) 1155-1161.

[11] K.A. Hawick, A. Leist, D. P. Playne, Parallel Graph Component Labelling with GPUs and CUDA, Parallel Computing 36 (2010) 655-678.

[12] O. Kalentev, A. Rai, S. Kemnitzb, R. Schneider, Connected component labeling on a 2D grid using CUDA, J. Parallel Distrib. Comput. 71 (2011) 615-620. 
[13] J. M. Kosterlitz, D. J. Thouless, Ordering, metastability and phase transitions in two-dimensional systems, J. Phys. C: Solid State Physics 6 (1973) 1181-1203.

[14] J. Hoshen, R. Kopelman, Percolation and cluster distribution. I. Cluster multiple labeling technique and critical concentration algorithm, Phys. Rev. B 14 (1976) 3438-3445.

[15] T. Aoki, S. Ogawa, A. Yamanaka, Multiple-GPU Scalability of Phase-Field Simulation for Dendritic Solidification, Prog. in Nucl. Sci. and Tech., 2 (2011) 639-642.

[16] T. Shimokawabe, T. Aoki, C. Muroi, J. Ishida, K. Kawano, T. Endo, A. Nukada, N. Maruyama, S. Matsuoka, An 80-Fold Speedup, 15.0 TFlops Full GPU Acceleration of Non-Hydrostatic Weather Model ASUCA Production Code, Proceedings of the 2010 ACM/IEEE International Conference for High Performance Computing, Networking, Storage and Analysis, November 13-19, 2010, pp. 1-11.

[17] T. Shimokawabe, T. Aoki, T. Takaki, T. Endo, A. Yamanaka, N. Maruyama, A. Nukada, S. Matsuoka, Peta-scale phase-field simulation for dendritic solidification on the TSUBAME 2.0 supercomputer, Proceedings of the 2011 ACM/IEEE conference on Supercomputing (SC'11), Seattle, WA, USA, Nov 2011.

[18] G. T. Barkema, T. MacFarland, Parallel simulation of the Ising model, Phys. Rev. E 50 (1994) 1623-1628.

[19] Y. Tomita, Y. Okabe, Probability-changing cluster algorithm for twodimensional XY and clock models for the probability-changing cluster algorithm, Phys. Rev. B 65 (2002) 184405.

[20] Y. Tomita, Y. Okabe, Finite-size scaling of correlation ratio and generalized scheme for the probability-changing cluster algorithm, Phys. Rev. B 66 (2002) 180401(R). 